\title{
Emergency Use of Convalescent Plasma: Perception of the Regulatory Framework from a Clinical Perspective
}

\author{
Patrick Möhnle $^{\mathrm{a}} \quad$ Andreas Humpe $^{\mathrm{a}}$ Markus Boeck $^{\mathrm{b}} \quad$ Stefanie Gruetzner $^{\mathrm{c}}$ \\ Holger Hackstein ${ }^{d}$ Robert Offner ${ }^{\mathrm{e}}$ Martin Hildebrandt ${ }^{\mathrm{a}}$ \\ aDivision of Transfusion Medicine, Cell Therapeutics and Haemostaseology, LMU Klinikum Großhadern, Munich, \\ Germany; ${ }^{b}$ Institute of Transfusion Medicine and Haemotherapy, University of Würzburg, Würzburg, Germany; \\ 'Department of Transfusion Medicine and Hemostaseology, University Hospital Augsburg, Augsburg, Germany;

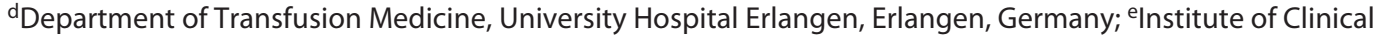 \\ Chemistry and Laboratory Medicine, Transfusion Medicine, University Hospital Regensburg, Regensburg, Germany
}

\section{Keywords}

Convalescent plasma $\cdot$ Regulation $\cdot$ Pandemic $\cdot$ Clinical use

\begin{abstract}
The pandemic spread of an infectious disease poses a plethora of challenges to society, clinicians, health care providers and regulating authorities. In order to mount a rapid response and to provide hope in a potentially catastrophic situation as the current COVID-19 pandemic, emergency plans, regulations and funding strategies have to be developed on regional, national and international levels. The speed needed to establish rapid response programs is challenged by the dynamics of the spread of the disease, the concurrent and competing development of different and potentially more effective treatment options, and not the least by regulatory uncertainty. Convalescent plasma, that is plasma collected from patients who have recovered from COVID-19 infections, has emerged as one of the first potential treatment options in the absence of drugs or vaccines with proven efficacy against SARS-CoV-2. The societal aspects of convalescent plasma and the public awareness gave an additional boost to the rapid employment of convalescent plasma donation platforms immediately after the SARS-CoV-2 outbreak. At the same time, uncertainty remains as to the efficacy of convalescent plasma. With evidence mostly limited to empirical reports, convalescent plasma has been used for decades for the prophylaxis and treatment of various infec-
\end{abstract}

karger@karger.com www.karger.com/tmh

Karger $\frac{1}{\%}$

BOPEN ACCESS
(C) 2021 The Author(s).

Published by S. Karger AG, Basel

This article is licensed under the Creative Commons Attribution 4.0 International License (CC BY) (http://www.karger.com/Services/ OpenAccessLicense). Usage, derivative works and distribution are permitted provided that proper credit is given to the author and the original publisher. tious diseases. Clinical trials have addressed different infectious agents, stages of disease, target groups of patients and yielded sometimes inconclusive results. The aim of this short review is to delineate the regulatory background for the emergency use of convalescent plasma in the USA, in the European Union and in Germany, and the transition to the setting of clinical trials. In addition, we describe observations made in the process of collecting COVID-19 convalescent plasma (herein referred to as (CP), and formulate proposals to further improve the framework for rapid responses in future emergency situations.

\footnotetext{
(c) 2021 The Author(s).

Published by S. Karger AG, Basel
}

\section{Introduction}

In the outbreak and rapid expansion of a viral infection, large groups of persons susceptible to the pathogen may become ill early in the event, prior to a detailed knowledge of the pathogen, the pathophysiology of the respective disease, and to the availability of effective vaccines and antiviral therapies.

An organized program to collect convalescent plasma or serum from disease survivors could provide a potentially valuable empirical intervention [1]. However, while the concept of convalescent plasma is theoretically feasible and convincing in general terms, valid data on the effectiveness and safety of its use in the context of a novel 
infectious threat need to be derived from randomized controlled clinical trials which generally require a minimum of time and may yield heterogeneous results [2]. The regulatory challenge, therefore, is to define and authorize emergency use under the constraints of limited evidence as well to promote a swift transition to standard pathways of clinical testing and potentially routine clinical use.

\section{Emergency Use of Convalescent Plasma: Definition} and Development

United States of America

In the USA, the term "emergency use" with regard to CCP appeared in April 2020 when the FDA issued a guidance document for the collection, shipment, clinical use and documentation of CCP for the treatment of hospitalized patients with COVID-19 [3]. In the absence of an approval for use by the FDA, CCP was classified as an investigational product under regulatory aspects.

On August 23, 2020, the FDA issued an emergency use authorization (EUA) for CCP for the treatment of hospitalized patients with COVID-19 [4]. A letter of authorization was needed for the emergency use. Outside this authorization, CCP would be regarded as an investigational product to be administered under different pathways: the traditional IND (investigational new drug) regulatory pathway, a single-patient IND for emergency use, or an intermediate-size population expanded access IND.

Only a few days later (August 28, 2020), the expanded access national program ended, terminating the use of investigational CCP that had been manufactured under less stringent conditions. Questions arose as to the use of CCP manufactured prior to the EUA and kept on stock that would not meet the new and more stringent requirements, including testing plasma donations for antiSARS-CoV-2 antibodies as a manufacturing step to determine suitability before release, using a test referenced in the EUA Letter of Authorization, as well as qualifying the unit as high titer CCP, based on the results of this testing.

On February 11, 2021, the FDA issued a revised guidance for industry with recommendations on donor eligibility and qualification, testing plasma for anti-SARSCoV-2 antibodies, and labeling. In awareness of the need to adapt to the new conditions, the FDA offered discretion with respect to the IND requirements until May 31, 2021, if certain conditions were met. Interestingly, these conditions address also the eligibility criteria for individuals who have received a COVID-19 vaccine or COVID-19 monoclonal antibody therapy to qualify for donation.

As such, the EUA was not implemented as a substitute to clinical trials; rather, CCP manufactured under the EUA was intended to facilitate the standardized use of
CCP in hospitalized patients with COVID-19, and complement the use of investigational CCP in clinical trials to fully answer the questions about the effectiveness of CCP for the treatment of COVID-19. Blood establishments would therefore collect CCP either under the conditions of EUA or as an investigational product under an IND.

\section{European Union}

The European Union (EU) competent authorities for blood and blood components, the European Centre for Disease Prevention and Control and the European Commission concurred in stating that CCP could be a viable option for the treatment of patients suffering from COVID-19. On April 8, 2020, a guidance document was published recommending that the use of CCP [5], as an immediately available experimental therapy with low risk, should be considered as an urgent priority and its outcome monitored. This recommendation was reinforced in June 2020 in a revised version of the guidance when further evidence was available. In particular, early data from 5,000 transfusions in hospitalized patients with COVID-19 in the USA in the FDA "expanded access" framework had proven a high level of safety [6]. These early data were confirmed by data based on 20,000 CCP transfusions [7]. Together with a meta-analysis by the European Centre for Disease Prevention and Control [2] and data from outside the EU, enough evidence was seen to further support the collection, clinical use and monitoring of CCP [5], to structure the efforts in the EU by the establishment of a common database in close collaboration with the European Blood Association, and to use an emergency support instrument (ESI) for the establishment of a European infrastructure for the collection of CCP in preparation for future pandemics. In the $\mathrm{EU}$, the regulation of $\mathrm{CCP}$ follows the regulation of plasma and other blood components (Directive 2002/98/EC [8]) unless the plasma is processed to other medicinal products subject to Directive 83/2001/EC. More stringent national rules than Directive 2002/98/EC may apply.

\section{Germany}

In Germany, blood components fall under the Medicinal Products Act (AMG, section 4, part 2 [9]). The AMG sets specific rules for the emergency use of medicines in catastrophic events (AMG, section 79, parts 1 and 5), granting the Federal Ministry of Health to "permit exceptions to the regulations laid down by this Act [...] if the necessary supply of medicinal products to the population would otherwise be seriously jeopardized and if a direct or indirect hazard by medicinal products to human health is not to be feared; in particular, regulations can be adopted to counter the spread of risks that might occur in 
reaction to the presumed or confirmed spread of pathogenic substances, toxins, chemicals or exposure to ionising radiation." In such a time of crisis, the Ministry of Health will allow the competent authorities to "permit, on a case-by-case basis, that medicinal products which are not authorized or registered for placing on the market within the purview of this Act:

1. may be placed on the market temporarily, and

2. may be introduced into the purview of this Act by way of derogation from section 73 sub-section 1 ,

in the event of a shortage of medicinal products necessary for the prevention or treatment of life-threatening diseases in the population or in the event of a dangerous communicable disease the spread of which calls for the provision of specific medicinal products immediately and in a quantity that considerably exceeds the norm."

In addition, the competent authorities may, on a caseby-case basis, also permit a temporary deviation from the authorization requirements or from other prohibitions contained in this Act. The assessment whether a shortage or a dangerous communicable disease exists or no longer exists is at the responsibility of the Federal Ministry of Health. Therefore, the permissions described above will end once the Ministry will declare the end of the crisis.

In 2015, the Working Group Blood at the German National Authority for Infectious Diseases, the Robert Koch Institute, issued recommendations on the use of convalescent plasma in the case of a pandemic [10], in the aftermath of the influenza pandemic spread 2009/2010. The regulatory environment for convalescent plasma was summarized as follows:

- convalescent plasma was defined as a serum and as a finished medicinal product, in accordance with the German AMG;

- a marketing authorization was not needed for directed use;

- at times of crisis, convalescent plasma could be applied without authorization, in accordance with chapter 2 of the Regulation on exemptions from the Medicinal Products Act for Civil protection, and chapter 79, parts 1 and 5, of the German AMG;

- the verification of the proof of concept for convalescent plasma should be exemplified in clinical trials;

- if clinical trials were successful, a convalescent plasma program should be activated and pursued under the auspices of a central and interdisciplinary steering group.

The regulation would exempt medicinal products needed in times of crisis also from European Regulations such as Council Regulation (EEC) No. 2309/93 of July 22, 1993 , laying down community procedures for the authorization and supervision of medicinal products for human and veterinary use and establishing a European Agency for the Evaluation of Medicinal Products. Again, national law would supersede EU regulations.
Convalescent Plasma: From Emergency Use to Clinical Trials

Convalescent plasma tested in clinical trials is defined as an investigational product and is dealt with under the rules of GCP-ICH and subjected to national or EU rules for clinical trials. Early on, competent authorities in the EU and in the USA have recommended CCP to be tested preferably in clinical trials $[3,11]$.

\section{United States of America}

The FDA has declared CCP to be an investigational medicinal product by definition, in the absence of an approval. Outside of the EUA, investigational CCP may be administered under the traditional IND regulatory pathway, a single-patient IND for emergency use, or an intermediate-size population expanded access IND [3]. It became apparent that the quality standards requested as part of the EUA led to specifications that were higher than possibly considered in some IND programs and other treatment scenarios. For instance, CCP collected prior to the EUA could have remained in inventory, and investigational CCP would have to be collected while operational changes were made to meet the new and more stringent requirements in the EUA. Considering these issues, the FDA offered to exercise temporary enforcement discretion regarding the IND requirements for the use of investigational CCP. The FDA intends to exercise this temporary enforcement discretion under certain circumstances, recommended the measurement of neutralizing antibody titers when available and set a limit of May 31, 2021, for this transition phase.

\section{European Union}

The European Commission mounted substantial effort to help Member States respond to the coronavirus pandemic by addressing various needs in a strategic and coordinated manner at a European level. The regulatory instrument used for this purpose, the ESI, is "based on the principle of solidarity and pools efforts and resources to quickly address shared strategic needs. The instrument helps mitigate the immediate consequences of the pandemic and anticipate the needs related to the recovery" [12]. Among the facets of ESI, purchase of rapid antigen tests and strengthening testing capacity in Member States, support for clinical trials for repurposed medicines and securing drug doses, the European Commission selected 24 projects to collect plasma from donors recovered from COVID-19 and to build preparedness for the rapid collection in future waves of the pandemic and also to establish an infrastructure in response to future pandemics.

With clinical trials still being regulated on a national level until Regulation 536/2014 will enter into force, the European Commission issued and updated guidance on $\mathrm{CCP}$ collection, testing, processing, storage and distribution [5]. Different from the FDA guidance on the EUA, 
details on investigational pathways are not addressed. However, it is "urgently recommended that patients receiving convalescent plasma are entered into a trial or are monitored through sharing of coded data on the EU public access platform described below. Convalescent plasma for use in an approved randomized or controlled clinical trial should be distributed according to the protocol of that trial and, where relevant, in compliance with national legislation" [5]. Also, a harmonized approach towards collection of data regarding the use of CCP, its safety and quality is strongly encouraged and defined in detail.

Serious adverse reaction and event notifications by hospitals to blood establishments are to be proactively reported to the competent authority and included in the annual EU serious adverse reaction and event reporting exercise to the European Commission, irrespective of the fact that the plasma has been transfused in a controlled clinical trial or an observational study.

\section{Germany}

In Germany, local and federal competent authorities engaged early on in the regulatory framework for the provision of CCP, collaborating closely with blood establishments to endorse the rapid establishment of donation programs. While the local competent authorities provided support in the process for permissions according to section 79 AMG (see above), the federal competent authority (Paul Ehrlich Institute) provided advice for clinical trials, definition of donor eligibility criteria and product specifications. A first trial on the use of CCP was granted authorization in April 2020, one week after submission of application [13]. As of May 28, 2021, at least 5 clinical trials have been registered in Germany (CAPSID, COMET, IPCO, RECOVER, Res-Q-HR). In Europe, 27 trials are listed as active and not recruiting, recruiting or completed (USA: 43 trials) [14]. Federal competent authority and ethics committees fostered and prioritized the authorization of clinical trials related to the treatment of COVID-19 infections in general. On a national basis, however, a framework of preparedness for clinical trials was not evident, including communication, coordination and central, application-free funding based on a study template. As a consequence, efforts to harmonize clinical trials on the use of CCP lacked conviction and appeared too slow to contribute substantially to the generation of knowledge, especially on efficacy to ascertain the usefulness of CCP manufacture and clinical application.

As in the USA, questions arose to the use of CCP manufactured and stored under permissions by local competent authorities, as these batches are manufactured neither under a license (section $13 \mathrm{AMG}$ ) nor according to an investigational medicinal product dossier. However, it was suggested that available stocks of CCP could be used as an investigational medicinal product by using a sec- ondary packaging specific for the respective trial and compliant with the labeling requirements laid down in annex 13 of the EU GMP guideline [15].

Programs outside the arena of clinical trials remain less defined. Regulatory uncertainty regarding the special manufacturing permissions became apparent when the collection, analysis and publication of data related to the donation came under scrutiny. Data collection and reporting are paramount to any structured effort to establish CCP donation programs. However, these formats would typically apply to licensed products and clinical trials [16]. Even more so, reporting of data related to the application (safety being pertinent to programs outside of clinical trials) would be looked upon as a research activity requiring an authorization of a clinical trial by the federal competent authority as well as a positive opinion from the sides of the ethics committee in charge.

Since the status of CCP manufactured under the emergency permission is not a licensed medicine it may only be applied either under the circumstances and conditions of single patient use ("individueller Heilversuch"), which is usually restricted to single or few treatments on a strictly individual basis, or under the conditions of a clinical trial. With the lack of clinical trials in the early phase of the pandemic, this restriction poses an additional challenge with regard to obtaining data on clinical efficacy and safety: the priority in single patient use is to optimize the outcome in the single patient in the lack of other treatment options and therefore opposes the gain of knowledge fundamental to clinical trials. Accordingly, data gained in single patient use can give little evidence on efficacy and safety.

\section{Convalescent Plasma: Challenges and Options}

Emergency situations such as a pandemic have to be dealt with in a structured, cooperative manner, in the face of public awareness, urgent medical need for treatment options and fear. The overwhelming numbers of responses to calls for plasma donation and the media coverage brought academic blood centers represented by the authors, other blood establishments and all institutions involved to the limits of their capacity. The lessons learned in this context include the following statements:

Cooperation and communication are essential. Close contact to the local and federal authorities was crucial in mounting a coordinated response, but even more important was a regular, transparent and trustful exchange among the blood centers, in this case the Bavarian academic blood centers. Collaboration allowed for the rapid establishment of common or similar documents for manufacture, testing and release, joint communication with authorities to define donation criteria, coding of products, mutual support in supply to patients, joint proposals of clinical trials and joint application in funding programs such as the ESI (see above). 
Structured programs for convalescent plasma donation and supply were missing. In their 2015 statement [10], the German Working Group Blood had proposed such structures to be established, but many suggestions had not received the deserved attention, on many levels. The European Commission recognized the need to provide support by funding networks and infrastructure of convalescent plasma donation, laying the basis for the availability of convalescent plasma in future pandemics. The proof of efficacy, however, is a distinguishing feature of randomized clinical trials. As there is no time to waste in emergency situations, a plethora of clinical trials that are too small or too late for success will be deleterious. Attempts to prove efficacy of convalescent plasma can only be successful through proper central coordination and communication to yield useful results as quickly as possible. Since convalescent plasma could be life-saving in a pandemic situation, such a concerted and centrally coordinated approach should be an essential component of rapid response programs. As for Germany, a central coordination, guidance and funding policy of an emergency clinical trial program should be in place, as a joint effort above and beyond state regulations as well as local and regional interests.

\section{Conclusion}

The safety of CCP has been proven. The efficacy may continue to be difficult to be proven, also because clinical observations outside clinical trials will continue to feed into the belief of efficacy, be it in general, be it in distinct subgroups of recipients.

In order to respond to a pandemic more effectively, knowledge on the efficacy of first-line approaches such as convalescent plasma has to be collected faster and in a coordinated manner. More stringent measures are needed to test convalescent plasma for efficacy in a set of centrally coordinated clinical trials. In our experience regulatory constraints and uncertainty, as well as the lack of quickly available funding pathways, have partially hin- dered a fast transformation of early clinical use to coordinated clinical trials. A comprehensible and straightforward regulatory network as well as the provision of easily accessible grants are needed for future pandemics. In addition, regulatory uncertainty regarding the use of data collected outside clinical trials should be dealt with proactively for future pandemics and emergency responses.

The provision of convalescent plasma has its time and its limitations. Structured programs and networks may allow for a strengthened preparedness for future pandemics, but also for a faster and more effective response, also in recognition of the donors' solidarity and altruism in the face of an unexpected and imminent threat to society.

\section{Acknowledgement}

The authors wish to acknowledge the support by the EU, DG Health, as part of their ESI.

\section{Conflict of Interest Statement}

The authors have no conflicts of interest to declare.

\section{Funding Sources}

Parts of the work leading to this manuscript have been funded by the EU, DG Health, as part of their ESI.

\section{Author Contributions}

All authors have contributed to the conception of the work, drafted or revised the manuscript critically for important intellectual content, given their final approval of the version to be published, and agreed to be accountable for all aspects of the work in ensuring that questions related to the accuracy or integrity of any part of the work are appropriately investigated and resolved.

\section{References}

1 WHO Blood Regulators Network. Position paper on use of convalescent plasma, serum or immune globulin concentrates as an element in response to an emerging virus. Available from: https://www.who.int/bloodproducts/brn/2017_BRN_PositionPaper_ConvalescentPlasma.pdf.

2 European Centre for Disease Prevention and Control (ECDC). Vaccines and treatment of COVID-19. Available from: https://www. ecdc.europa.eu/en/covid-19/latest-evidence/ vaccines-and-treatment.

3 US Food and Drug Administration . Recommendations for investigational COVID-19 convalescent plasma [Internet] [cited 2021 Feb 27]. Available from: https://www.fda. gov/vaccines-blood-biologics/investigational-new-drug-ind-or-device-exemption-ideprocess-cber/recommendations-investigational-covid-19-convalescent-plasma.

4 US Food and Drug Administration. Emergency use authorization [Internet] [cited 2021 Feb 27]. Available from: https://www.fda. gov/media/141477/download.

5 An EU programme of COVID-19 convalescent plasma collection and transfusion. Guidance on collection, testing, processing, storage, distribution and monitored use. Available at: https://ec.europa.eu/ health/sites/health/files/blood_tissues_organs/docs/guidance_plasma_covid19_ en.pdf.

6 Joyner M, Wright RS, Fairweather D, Senefeld J, Bruno K, Klassen S, et al. Early safety indicators of COVID-19 convalescent plasma in 5,000 patients. medRxiv. 2020. 2020.05.12.20099879.

7 Joyner MJ, Bruno KA, Klassen SA, Kunze KL, Johnson PW, Lesser ER, et al. Safety update: COVID-19 convalescent plasma in 20,000 hospitalized patients. Mayo Clin Proc. 2020 Sep;95(9):1888-97. 
8 Directive 2002/98/EC of the European Parliament and of the Council of 27 January 2003 setting standards of quality and safety for the collection, testing, processing, storage and distribution of human blood and blood components and amending Directive 2001/83/EC.

9 Medicinal Products Act in the version published on 12 December 2005 (Federal Law Gazette [BGBl]). Part I, p. 3394, last amended by Article 11 of the Act of 6 May 2019 (Federal Law Gazette I, p. 646).

10 Stellungnahme zur Gewinnung und Nutzung von Rekonvaleszentenplasma (RKP) als Therapieoption bei Ausbrüchen schwerer Infektionen. Bundesgesundheitsblatt. 2015;58: 1371-7.
11 https://www.pei.de/DE/newsroom/hpmeldungen/2020/200407-empfehlung-peicovid-19-rekonvaleszentenplasma-rkp.html; jsessionid=0988A5E3E64A12011927048 $1 \mathrm{D} 0948945$.

12 Emergency support instrument. Available from: https://ec.europa.eu/info/live-worktravel-eu/coronavirus-response/emergencysupport-instrument_en\#treatments.

13 https://www.pei.de/EN/newsroom/press-releases/year/2020/07-pei-approves-first-covid-19-therapy-study-with-convalescent-plasma.html;jsessionid=8D95B75D92205D1478 C1F732BF15A3EE.intranet241.

14 https://clinicaltrials.gov/ct2/results/map?rec $\mathrm{rs}=$ ade\&cond $=$ convalescent + plasma $\&$ map $=$.
15 EudraLex. The rules governing medicinal products in the European Union volume 4. EU guidelines to good manufacturing practice medicinal products for human and veterinary use. Annex 13: Investigational medicinal products. Brussels, February 3, 2010 Available from: https://ec.europa.eu/health/ sites/health/files/files/eudralex/vol4/2009_06_annex13.pdf.

16 E6(R2) good clinical practice: integrated addendum to ICH E6(R1) guidance for industry. Available from: https://www.fda.gov/ drugs/guidance-compliance-regulatory-information/guidances-drugs. 